\title{
Opinion
}

\section{Archiving Primary Data: Solutions for Long-Term Studies}

James A. Mills, ${ }^{1, \#, \star}$ Céline Teplitsky, ${ }^{2, \#, \star}$ Beatriz Arroyo, ${ }^{3}$ Anne Charmantier, ${ }^{4}$ Peter. H. Becker, ${ }^{5}$ Tim R. Birkhead, ${ }^{6}$ Pierre Bize, ${ }^{7}$ Daniel T. Blumstein, ${ }^{8}$ Christophe Bonenfant, ${ }^{9}$ Stan Boutin, ${ }^{10}$ Andrey Bushuev, ${ }^{11}$ Emmanuelle Cam, ${ }^{12}$ Andrew Cockburn, ${ }^{13}$ Steeve D. Côté, ${ }^{14}$ John C. Coulson, ${ }^{15}$ Francis Daunt, ${ }^{16}$ Niels J. Dingemanse, ${ }^{17,18}$ Blandine Doligez, ${ }^{9}$ Hugh Drummond, ${ }^{19}$ Richard H.M. Espie, ${ }^{20}$ Marco Festa-Bianchet, ${ }^{21}$ Francesca Frentiu, ${ }^{22}$ John W. Fitzpatrick, ${ }^{23}$ Robert W. Furness, ${ }^{24}$ Dany Garant, ${ }^{21}$ Gilles Gauthier, ${ }^{14}$ Peter R. Grant, ${ }^{25}$ Michael Griesser, ${ }^{26}$ Lars Gustafsson, ${ }^{27}$ Bengt Hansson, ${ }^{28}$ Michael P. Harris, ${ }^{16}$ Frédéric Jiguet, ${ }^{29}$ Petter Kjellander, ${ }^{30}$ Erkki Korpimäki, ${ }^{31}$ Charles J. Krebs, ${ }^{32}$ Luc Lens, ${ }^{33}$ John D.C. Linnell, ${ }^{34}$ Matthew Low, ${ }^{35}$ Andrew McAdam, ${ }^{36}$ Antoni Margalida, ${ }^{37}$ Juha Merilä, ${ }^{38}$ Anders P. Møller, ${ }^{39}$ Shinichi Nakagawa, ${ }^{40}$ Jan-Åke Nilsson, ${ }^{27}$ lan C.T. Nisbet, ${ }^{41}$ Arie J. van Noordwijk, ${ }^{42}$ Daniel Oro, ${ }^{43}$ Tomas Pärt, ${ }^{35}$ Fanie Pelletier, ${ }^{21}$ Jaime Potti, ${ }^{44}$ Benoit Pujol, ${ }^{13}$ Denis Réale, ${ }^{45}$ Robert F. Rockwell, ${ }^{46}$ Yan Ropert-Coudert, ${ }^{47}$ Alexandre Roulin, ${ }^{48}$ James S. Sedinger, ${ }^{49}$ Jon E. Swenson, ${ }^{50}$ Christophe Thébaud, ${ }^{12}$ Marcel E. Visser, ${ }^{42}$ Sarah Wanless, ${ }^{16}$ David F. Westneat, ${ }^{51}$ Alastair J. Wilson, ${ }^{52}$ and Andreas Zedrosser ${ }^{53}$

The recent trend for journals to require open access to primary data included in publications has been embraced by many biologists, but has caused apprehension amongst researchers engaged in long-term ecological and evolutionary studies. A worldwide survey of 73 principal investigators (PIs) with long-term studies revealed positive attitudes towards sharing data with the agreement or involvement of the $\mathrm{PI}$, and $93 \%$ of PIs have historically shared data. Only $8 \%$ were in favor of uncontrolled, open access to primary data while $63 \%$ expressed serious concern. We present here their viewpoint on an issue that can have non-trivial scientific consequences. We discuss potential costs of public data archiving and provide possible solutions to meet the needs of journals and researchers. 


\section{Long-Term Data Sharing}

Several funding agencies, international regulatory bodies, and many major ecological and evolutionary journals now require raw or primary data to be deposited in a permanent open access archive, such as Dryad or TreeBASE, as a condition for funding or publication. The data must be in sufficient detail to allow the analyses in the paper to be replicated. The rationale for open archiving is that archived data are available to posterity when studies are completed, for error-checking, for use in new studies, or for future meta-analysis [1]. In addition it has been argued that the policy would benefit data providers by increasing their citation index through citations by papers with new analyses [1,2].

Although it is claimed that over $95 \%$ of scientists in evolution and ecology believe that data should be publicly archived [1], mandatory public data archiving (PDA) is raising many issues in the scientific community as evidenced by debates on websites, in blogs, and in publications ([2-9]; McGlynn, T. (2014) I own my data, until I don't. http://smallpondscience.com/2014/03/ 03/i-own-my-data-until-i-dont/). We focus on the perspective from long-term individual-based studies of wild populations that often span several decades.

Short and long-term ecological studies differ in several important aspects. For example, in the former, data tend to be collected over a short period of time leading to one or two publications, and once published the data in these papers become less valuable to the collector and can be more useful to others with different perspectives or analytical skills. By contrast, in studies that have followed individuals over their lifetimes, a great deal of crucial information is assessed from derived metrics (e.g., survival, lifetime reproductive success) that can only be estimated after many years of fieldwork. Therefore, much value can remain in the primary data even after some of the initial questions have been answered.

Long-term studies are rare and have great scientific value because many important questions in ecology and evolutionary biology can only be answered from the life-histories of recognizable individuals [10]. A detailed analysis of the importance of individual-based studies has been documented elsewhere [10], but a few examples are given in Box 1.

While group discussions and blog posts on PDA-related issues have been flourishing, little is formally known and published about the position and concerns of people collecting long-term data. To fill this gap, a survey was conducted to learn their perspectives, and if current data requirements were perceived as being problematic, to identify potential alternative data-sharing policies that could be acceptable to the journals, the scientific community, and the Pls.

\section{The Survey}

To obtain the opinions of scientists with individual-based longitudinal data, a worldwide survey was sent to 146 Pls of long-term research projects. Responses were received from 73 Pls

\section{Box 1. Ecological and Evolutionary Importance of Long-Term Studies}

Studies that have followed individuals throughout their lives have yielded important insights into demographic and lifehistory parameters of wild populations which cannot be obtained from cross-sectional programs. They have provided details on the impact of age, individual quality, status of pair-bond and food abundance on aspects of breeding, recruitment and survival [11-24]; cost of breeding [25]; inbreeding [26-28]; senescence [29-30]; mate choice [31-32]; carry-over and transgenerational effects [33-37] and lifetime reproduction [38-39]. Studies that assembled pedigrees from observational and or molecular techniques have enabled widespread application of quantitative genetic methods to questions in evolutionary ecology, such as maintenance of genetic variance, inheritance of fitness components and the relative contribution of plasticity and micro-evolutionary processes to phenotypic change [40-47]. Importantly, long-term studies have also been the basis for understanding the impact of climate change [48-51], habitat loss and natural resource overexploitation [52]. To address many of the aspects or issues listed above, researchers must have access to a marked age-structured and pedigreed study population which can take a minimum of 10 to 20 years of monitoring to establish, depending on the generation time of the species.

\section{Trends}

Public data archiving is the archiving of primary data used in publications so that they can be preserved and made accessible to all online.

Public data archiving is increasingly required by journals. However, the costs of public data archiving might be underestimated, in particular with respect to long-term studies.

Long-term studies have been responsible for the answers to many important questions in evolution and ecology which could only be answered through following the life-histories of individuals for decades.

Several papers have been published in favor of public data archiving, but a more balanced viewpoint is necessary to allow a discussion to emerge on a code of ethics and ways to preserve and protect the data, encourage the initiation and continuation of long-term studies, and meet the requirements of the whole scientific community.

${ }^{1} 10527$ A Skyline Drive, Corning, NY 14830, USA

2Département Ecologie et Gestion de la Biodiversité, UMR 7204 CNRS/ MNHN/UPMC, Muséum National d'Histoire Naturelle, Paris, France ${ }^{3}$ Instituto de Investigacion en Recursos Cinegeticos (IREC) (CSICUCLM-JCCM), Ronda de Toledo $s / n$, 13005 Ciudad, Real, Spain

${ }^{4}$ Centre d'Ecologie Fonctionnelle et Evolutive UMR 5175, Campus CNRS, 1919 Route de Mende, 34293 Montpellier CEDEX 5, France ${ }^{5}$ Institute of Avian Research, 'Vogelwarte Helgoland', An der Vogelwarte 21 D26386 Wilhelmshaven, Germany

${ }^{6}$ Department of Animal and Plant Sciences, University of Sheffield, Sheffield, UK

${ }^{7}$ Institute of Biological and

Environmental Sciences, University of

Aberdeen, Aberdeen, UK

${ }^{8}$ Department of Ecology and Evolutionary Biology, University of California, 621 Young Drive South, Los Angeles, CA 90095-1606, USA ${ }^{9}$ CNRS, Université Lyon 1, Université de Lyon, UMR 5558, Laboratoire Biométrie et Biologie Évolutive, 43 boulevard du 11 Novembre 1918, 69622 Villeurbanne CEDEX, France ${ }^{10}$ Department of Biological Sciences, University of Alberta, Edmonton, 


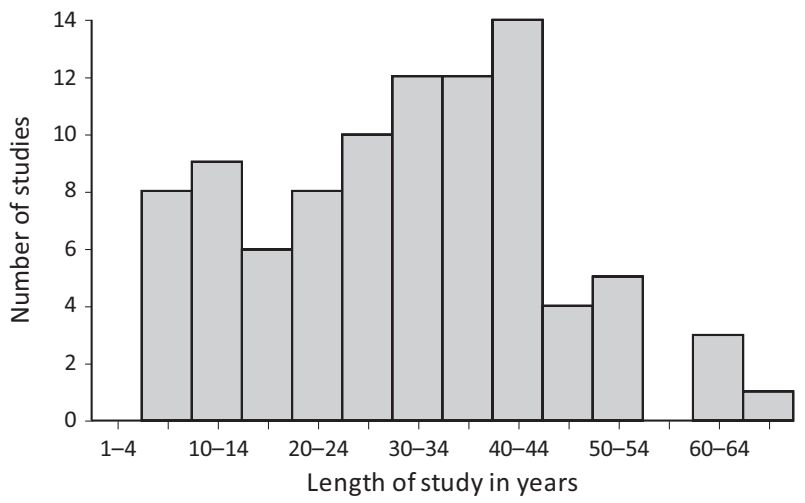

Trends in Ecology \& Evolution

working on 59 bird studies, 13 mammalian studies, and 1 plant study. The 92 projects (some Pls have several projects) range in duration from 5 to 68 years (Figure 1), with $55 \%$ collecting data for more than 30 years. Thirty-five percent of researchers were required to archive data used in a publication by their current funding agency and $19 \%$ by their institution. Eight researchers were required to deposit data by both; therefore 59\% were not required to archive their data. There was diversity of opinion among Pls about data archiving, but some strong points of consensus emerged. This paper synthesizes the views of all respondents, many of whom have made important contributions to ecology and evolution.

The survey revealed that virtually all Pls were in favor of data sharing with the agreement or involvement of the PI. Historically, 93\% of the respondents have shared their data when asked and $80 \%$ have collaborated in meta-analyses. In the 1960s to 1970 s publications using longitudinal data often involved only one or two authors. However, over the past two decades studies have become more complex and collaborative, with studies commonly involving collaboration among biologists with expertise in a variety of disciplines.

Overall, 63\% of Pls were against PDA as currently required. This contrasts with a previous survey of ecological and evolutionary biologists that reported that 95\% were in favor of PDA [1]. Among the $36 \%$ of respondents in favor of open-access data-archiving in this survey, only six ( $8 \%$ of 73 ) were in favor of unconditional data archiving. The reasons given by Pls in favor of PDA were similar to those advocated by the archiving journals. By contrast, $91 \%$ of Pls supported data sharing when clear rules for data access were in place. These rules could include (i) coauthorship or at least acknowledgment, depending on the level of PI involvement; (ii) no overlap with current projects, particularly projects conducted by students or postdoctoral fellows; and (iii) an agreement that the data go no further than the person to whom they are entrusted.

\section{General Concerns about PDA}

The main issues about archiving were centered on what data would be archived and to whom access would be given, as detailed below. However, these concerns are so strong that $41 \%$ of respondents said that they have avoided publishing in journals that require data be deposited in open-access archives. Furthermore, $53 \%$ intend to avoid publishing in them in the future and, for those who published a major paper involving long-term data early in their careers, $63 \%$ indicated that they would not have submitted it to any journal that required data archiving. Avoiding publishing in a high-impact journal can have major consequences in terms of career advancement, and could potentially reduce the prospects of obtaining future financial support; therefore the decision would not be taken lightly.
Alberta T6G 2E9, Canada

${ }^{11}$ Department of Vertebrate Zoology, Faculty of Biology, Lomonosov Moscow State University, Leninskie Gory 1/12, 119234 Moscow, Russia ${ }^{12}$ UMR 5174 EDB Laboratoire Évolution et Diversité Biologique, CNRS, ENFA, Université Toulouse 3 Paul Sabatier, 31062 Toulouse CEDEX 9, France

${ }^{13}$ Department of Evolution, Ecology and Genetics, Research School of Biology, The Australian National University, Canberra, ACT, Australia ${ }^{14}$ Département de Biologie and Centre d'Etudes Nordiques, Université Laval, 1045 avenue de la Médecine, Québec G1V 0A6, Canada

${ }^{15} 29$ St Mary's Close, Shincliffe, Durham DH1 2ND, UK

${ }^{16}$ Centre for Ecology and Hydrology,

Bush Estate, Penicuik, EH26 0QB UK

${ }^{17}$ Behavioural Ecology, Department of Biology, Ludwig-Maximilians University of Munich, PlaneggMartinsried, Germany

${ }^{18}$ Evolutionary Ecology of Variation Research Group, Max Planck Institute for Ornithology, Seewiesen, Germany ${ }^{19}$ Departamento de Ecología Evolutiva, Instituto de Ecología, Universidad Nacional Autónoma de México, AP 70-275, México DF 04510, México ${ }^{20}$ Technical Resource Branch,

Saskatchewan Ministry of

Environment, 3211 Albert Street, Regina, Saskatchewan, S4S 5W6, Canada

${ }^{21}$ Département de Biologie, Université de Sherbrooke, 2500 Boulevard de I'Université, Sherbrooke, Québec J1K 2R1, Canada

${ }^{22}$ School of Biomedical Sciences and Institute of Health and Biomedical Innovation, Queensland University of Technology, Kelvin Grove, QLD 4059 Australia

${ }^{23}$ Cornell Lab of Ornithology, 159 Sapsucker Woods Road, Ithaca, NY 14850, USA

${ }^{24}$ Graham Kerr Building, University of Glasgow, Glasgow G12 8QQ, UK ${ }^{25}$ Department of Ecology and Evolutionary Biology, Princeton University, Princeton, NJ 08544-1003, USA

${ }^{26}$ Anthropological Institute and

Museum, University of Zürich, Zürich, Switzerland

${ }^{27}$ Department of Animal Ecology, Evolutionary Biology Center, Uppsala University, Uppsala, Sweden ${ }^{28}$ Department of Biology, Lund University, Ecology Building, 223 62, Lund, Sweden

${ }^{29}$ CESCO, UMR7204 Sorbonne Universités-MNHN-CNRS-UPMC, CP51, 55 Rue Buffon, 75005 Paris, France 
In discussions among the survey respondents, it was suggested that the design and data collection of a long-term study constitute a research infrastructure that is the foundation of the publications which form the lifework and careers of researchers, PhD students, and postdoctoral fellows who work on these programs. The analogy can be made to experimental infrastructures which involve the construction of an apparatus that takes years, or sometimes decades, and requires numerous grant applications, institutional support, and deferred publication effort, all of which involve significant risk, but potentially have profound scientific value, both pure and applied. Developing the infrastructure is a necessary prerequisite for project completion. In this case it would not be reasonable for other scientists to have immediate access to the fruits of the inventor's labors. Furthermore, compulsory and unrestricted open access to the apparatus would provide a strong disincentive to making the initial infrastructural investment. The same case can be made for long-term ecological studies.

\section{Specific Concerns from Long-Term Researchers about PDA}

Several concerns about the costs of PDA for researchers and the scientific community were addressed previously [5]. We add here the perspective of Pls with long-term studies. Three major concerns were identified during the survey.

\section{Potential Costs to Science}

Flawed Science. A major cost would be flawed science resulting from a lack of understanding of the database or the biological system. Open access to long-term data might not allow full understanding of all the subtle contexts, nuances, and issues involved in the biological system and the structure of the database from which the long-term data are collected. It has been argued that if method sections are sufficiently detailed, misunderstanding the system should not be a major source of error [Coulson, T. and Sheldon, B. (2014) Archive your data! Animal ecology in focus. https://journalofanimalecology.wordpress.com/2014/11/21/ archive-your-data/]. However, not all of the complexities of the biological system can be detailed in a method section without making a paper unwieldy. Hence, without the involvement of the PI, crucial contextual information is likely to be lost under open access, leading to the potential for erroneous assumptions and interpretations which could add to the growing retraction rate in scientific journals [53]. For example, although it was not included as a question, three respondents of the survey indicated that on four occasions their data have been misinterpreted in publications and, once published, errors or misinterpretations are hard to remove.

\section{More Time Spent on Redundant Activities}

A potential cost would be simultaneous testing of the same idea on the data. In some cases, hypotheses might have been already investigated but not published by the Pls because they were inconclusive. In addition, the cost of monitoring publications that used PDA and writing replies would be borne by the researcher with long-term data and not by the scientific community. These do not seem to be a productive use of research investment.

\section{Fewer Long-Term Studies}

Open-access archiving could reduce the incentives for carrying out long-term studies and would likely result in researchers suspending ongoing studies and declining to undertake new ones. This is predicted by the producer-scrounger game theory [54] where the producer spends time and energy to develop a resource but is unable to monopolize it, thereby creating opportunities for the resource to be exploited by scrounger(s). Over time, as the scrounger strategy increases, the resource decreases. In theory, the fitness of the producer and the scrounger decrease because at some point there are no more resources to scrounge because no more resources are being produced $[55,56]$.
${ }^{30}$ Grimso Wildlife Research Station, Department of Ecology, Swedish University of Agricultural Sciences (SLU) 73091, Riddarhyttan, Sweden ${ }^{31}$ Section of Ecology, Department of Biology, University of Turku, 20014 Turku, Finland

${ }^{32}$ Department of Zoology, University of British Columbia, Vancouver, British Columbia V6T 1Z4, Canada

${ }^{33}$ Terrestrial Ecology Unit, Department of Biology, Ghent University, Ledeganckstraat 35, 9000 Gent, Belgium

${ }^{34}$ Norwegian Institute for Nature

Research, PO Box 5685 Sluppen, 7485 Trondheim, Norway

${ }^{35}$ Department of Ecology, Swedish University of Agricultural Sciences, 75007 Uppsala, Sweden

${ }^{36}$ Department of Integrative Biology, University of Guelph, Guelph, Ontario, N1G 2W1, Canada

${ }^{37}$ Faculty of Life Sciences and Engineering, University of Lleida, 25198 Lleida, Spain

${ }^{38}$ Ecological Genetics Research Unit, Department of Biosciences, PO Box 65 (Biocenter 3, Viikinkaari 1), University of Helsinki, 00014 Helskinki, Finland

${ }^{39}$ Laboratoire Ecologie, Systématique et Evolution, Equipe Diversité, Ecologie et Evolution Microbiennes, Bâtiment 362, 91405 Orsay CEDEX, France

${ }^{40}$ Evolution and Ecology Research

Centre and School of Biological, Earth and Environmental Sciences,

University of New South Wales,

Sydney, Australia

${ }^{41}$ I.C.T. Nisbet and Company, 150

Alder Lane, North Falmouth, MA 02556, USA

${ }^{42}$ Department of Animal Ecology, Netherlands Institute of Ecology (NIOO-KNAW), PO Box 50, 6700 AB Wageningen, The Netherlands

${ }^{43}$ Institut Mediterrani d'Estudis Avançats IMEDEA (CSIC-UIB), Miquel

Marques 21, 07190 Esporles,

Mallorca, Spain

${ }^{44}$ Departamento de Ecologia Evolutiva,

Estación Biológica de Doñana-CSIC,

Av. Américo Vespucio s/n, 41092 Seville, Spain

${ }^{45}$ Département des Sciences

Biologiques, Université du Québec A Montréal, CP 8888 Cuccursale Centre

Ville, Montréal, Québec H3C 3P8, Canada

${ }^{46}$ Vertebrate Zoology, American Museum of Natural History, New York, NY 10024 USA

${ }^{47}$ Institut Pluridisciplinaire Hubert Curien, CNRS UMR7178, 23 rue Becquerel 67087 Strasbourg, France

${ }^{48}$ Department of Ecology and Evolution, University of Lausanne, 


\section{Less Collaboration}

New collaborations are extremely valuable to make the most of the data, but comparative analyses and meta-analysis among long-term studies would likely suffer because Pls might decline to participate if they are required to archive their data.

\section{Research Funding}

Several financial issues have been overlooked by the advocates of PDA. Archiving mutualizes the benefits, but not the costs of long-term studies, because there is no cost to the person accessing the data. This might be a sustainable model when recurrent funding is available, but not when funding is granted on a per project basis. In addition, PDA could incur some new costs for long-term studies because Dryad, for example, has required extra payment for large datasets. Researchers with scarce funding might not be able to absorb this additional cost. Maintaining constant funding is a crucial issue for long-term studies to avoid fatal gaps in the data $[10,57]$, contrasting once again with short-term studies that can be restarted at a later time. Long-term studies of all durations experienced difficulties with funding (Figure 2) because only $33 \%$ were fully funded in all years, with the remainder having funding gaps varying in duration from 1 to 19 years (Figure 2). To maintain funding, Pls with long-term projects must keep identifying new uses of the data to obtain short-term funding because recurrent funding is essentially nonexistent [10]. Therefore, PDA could lead to a loss of funding opportunities if data for their next project are routinely mined by other researchers.

\section{Student Experience and Training}

A major contribution of long-term studies is that they often provide training to $\mathrm{PhD}$ and other postgraduate students and postdoctoral fellows. The Pls that responded to the survey reported that, from their 92 projects, 630 PhDs were awarded (Figure 3A), and 658 postgraduates and 257 postdoctoral fellows participated, for a total of 1545 trainees. This represents a substantial contribution to the training and development of the ecological and evolutionary biology research community. Survey respondents expressed a particular concern that PDA would negatively impact upon this important feature of long-term studies because negotiations take place among study participants before the onset of new research areas (such as MSc and PhD thesis or postdoctoral research projects) to avoid overlap. Such planning is undermined if outsiders are entirely free to work with available data from long-term studies without taking ongoing and planned analyses by insiders into account. The risk is especially strong for PhD students because part of their training involves courses, and they need more time to complete the research project and publish papers than do senior researchers.

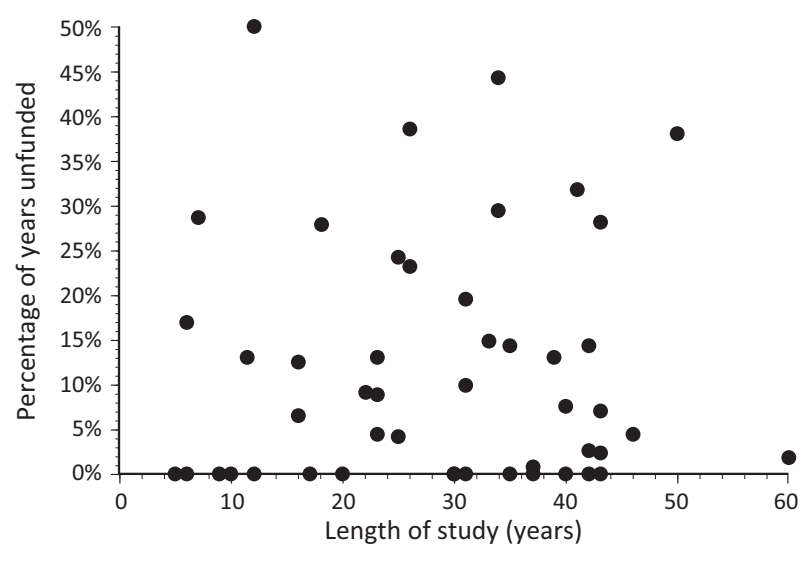

Figure 2. Duration of the Study and the Percentage of Years Unfunded.
Lausanne, Switzerland
${ }^{49}$ Department of Natural Resources and Environmental Science, University of Nevada Reno, Reno NV 89512, USA

${ }^{50}$ Department of Ecology and Natural Resource Management, Norwegian University of Life Sciences, PO Box 5003, 1432 Ås, and Norway and Norwegian Institute for Nature Research, PO Box 5685 Sluppen, 7485 Trondheim, Norway

${ }^{51}$ Department of Biology, Center for Ecology, Evolution, and Behavior, University of Kentucky, Lexington, KY, USA

${ }^{52}$ Centre for Ecology and Conservation, College of Life and Environmental Sciences, University of Exeter, Cornwall Campus, Penryn TR10 9EZ, UK

${ }^{53}$ Faculty of Arts and Sciences, Department of Environmental and Health Studies, Telemark University College, 3800 Bø i Telemark, Norway
*Correspondence: dmills@stny.rr.com (J.A. Mills), teplitsky@mnhn.fr (C. Teplitsky).

\#J.A.M. and C.T. contributed equally. 


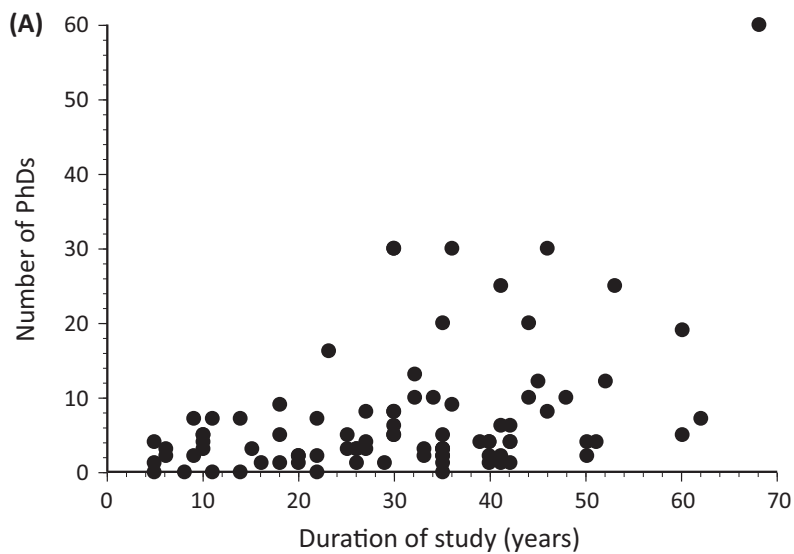

Figure 3. (A) The Total Number of PhD Students in Relation to the Duration of Research Programs. (B) The Number of Papers Produced in Relation the Duration of the Study.

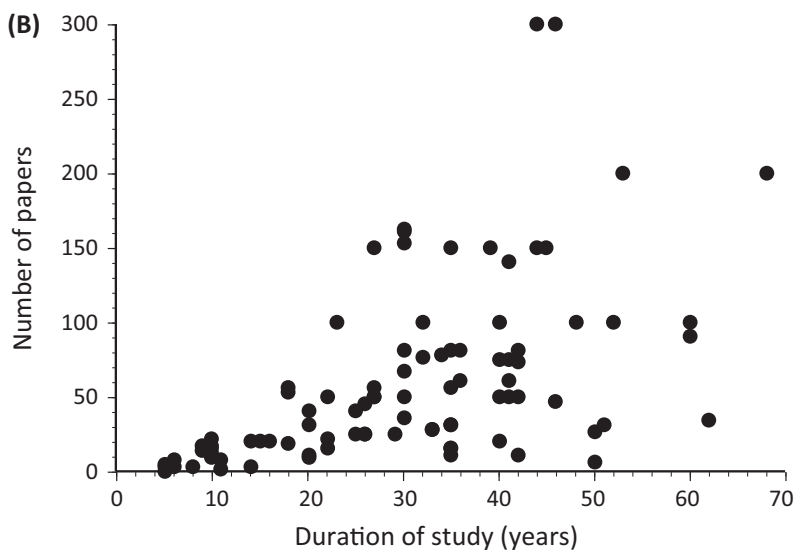

Trends in Ecology \& Evolution

\section{Possible Solutions}

The verification of results is a very important requirement by journals; however, the costs of mandatory archiving of data by ongoing long-term projects could outweigh the expected benefits. Having imposed a requirement for PDA, journals are asking researchers to give up rights to what many consider to be their intellectual property. In fact, some scientists are considering copywriting their data. Journals are rightly vigilant in combating plagiarism and copyright infringement; it would be appropriate for journals to be equally vigilant in respecting and protecting the scientists' data.

A resolution to this conflict would benefit scientific progress; high-quality long-term studies have been responsible for a disproportionate number of publications in journals with the highest impact factors [10]. Many of the 5378 papers from 90 studies in this survey (Figure 3B) were published in prestigious journals that now require PDA. To initiate a discussion about how resolution might be achieved, we suggest six potential solutions.

Promoting Collaboration

Opportunities for collaboration that provide added-value to science and communication between data generators and potential users should be encouraged [5] rather than compulsory archiving. Most survey respondents see collaborations as the most satisfactory route to data 
sharing. For substantial requests, the original researcher can expect and deserve coauthorship. To promote better use of data and collaboration with Pls, a website could be created referencing long-term studies with information such as species, duration of study, location, traits measured, and protocols used.

\section{Providing Primary Data on a Confidential Basis}

A solution that would satisfy most Pls would be to provide tabulated summary data initially, and if those data were insufficient for editors to evaluate a submitted paper, primary data could be provided on a confidential basis. After the review process, the data could be destroyed and would not be available to be used for any other purpose. Once the paper is published, people who want to use the data could contact the Pls of the long-term study for additional data. As the survey has shown, 93\% of the respondents have indicated that they have supplied data on request. For example, researchers have used summary data from the 40 year study on Darwin's finches $[44,58]$ by Peter and Rosemary Grant which was deposited in Dryad, and raw data have been supplied to four others upon request.

\section{Providing a Longer Embargo}

Some journals have indicated a willingness to embargo the data for a period of 1-5 years from publication, allowing the original researcher time to complete any related papers. This can reduce concerns in the case of smaller datasets from which only a limited number of questions can be answered. However, this is unlikely to solve the problem for long-term datasets, from which many questions can be addressed from different perspectives and over differing lengths of time.

For active long-term studies (i.e., with ongoing data collection) a minimum of 10-15 years might be considered more appropriate [5]. By comparison, pharmaceutical companies have a 20 year patent to recoup their investment. A similar argument could be made for the decades of research by long-term project scientists [McGlynn, T. (2014) I own my data, until I don't. http://smallpondscience.com/2014/03/03/i-own-my-data-until-i-dont/]. Furthermore, a longer embargo would encourage data users to contact the PIs for rapid access to the most up-to-date version of the database, thereby encouraging collaboration. For non-active studies where data collection has ended, the case for an earlier release is stronger.

\section{Depositing Data on Institutional Servers}

Centralizing the data in a single database in one location will prevent fragmentation of data on different archiving sites and ensure that the data are completely secure and up to date. Data could be archived on institutional servers, and the institution and its staff could control access and determine if collaboration is appropriate. An example of an effective approach to the management of archived data held by institutions is practiced by The Netherlands Institute of Ecology where people can request the data, and data extraction is carried out by members of the Institute, provided that the applicant will use the data for a well-described project, commit to not sharing the data with others, and offer coauthorship to the collector if the data forms an essential part of the publication. Another example of effective use of institutional servers is the Archibold Biological Station in Florida. Such institutional databases also allow the preservation of data and their accessibility after the PI retires [10].

Increasing Notification and Communication

If online archiving should be preferred for the physical safety of data, two improvements to present practices could be made. First, as the survey demonstrated, Pls are concerned with inappropriate use of data and overlap with ongoing or future projects of their own. A clear policy should be implemented by journals concerning conflicts of interest between the researchers collecting and organizing the data, and those who would use the data. For example, there are 
currently no binding protocols or codes of conduct covering the presentation of, or access to, complex data that underpin analyses in publications. A process with guidelines should be established by journals to ensure that Pls are aware of potential studies and are satisfied with a paper based on the data they generated before the review process.

A possibility would be to implement data-tracking, allowing data collectors to obtain information on who is using the data and why. For example, any request for data to the Climate Change, Agriculture, and Food Security Data Portal triggers an email to be sent to the PI who deposited the data. Journals should have a rule that no paper is considered where the data users have not corresponded with the data owners and included appropriate acknowledgement of the source of the data within the paper. A rule set by journals would have a great deal of clout with data users. Data tracking would also allow the PI to be systematically asked to review papers based on their data. Another option would be to send an email to the PI every year asking whether they wish the data to be private or open access.

\section{Concluding Remarks}

Long-term studies currently generate science with high impact in all major fields of biology. These longitudinal studies began during an era when PDA did not exist. Although we agree that it is essential to archive data so that they are not lost to science, a key concern is that recently introduced data-archiving regimes combined with difficulty in obtaining continuous financial support will be a disincentive both for the initiation of long-term studies, and for maintenance of ongoing studies. It would be appropriate for journals and data-archiving institutions to enter into a dialog with researchers about how best to meet the objectives of data archiving while allowing valuable long-term studies to thrive.

Specifically, we recommend the development of a formal code of conduct which respects the data generated through long-term studies, and (i) allow tabulated summaries to be provided in the first instance backed up by the confidential submission of primary data if required by editors, (ii) encourage collaborative research with the data collector by people wishing to use the data, (iii) extend embargoes on the use of archived data [5], (iv) consider allowing archiving on institutional servers rather than open-access servers, and (iv) develop enforceable procedures that enable the researcher to be contacted when someone wishes to access primary data. Through these modifications, a compromise could be crafted that provides an advantage to the scientific community, journals, and researchers generating long-term data, as well as benefiting science.

\section{Acknowledgments}

We wish to thank all of the principal investigators who provided information on their long-term studies. C.T. was funded by the French Agence Nationale de la Recherche (grant ANR-12-ADAP-0006). Many thanks to Sandra Hamel, Susan Alberts, and Walt Koenig for constructive comments. Deborah Mills assisted in the analysis and editing the manuscript.

\section{References}

1. Whitlock, M.C. et al. (2010) Data archiving. Am. Nat. 175, 145-146

2. Piwowar, H.A. and Vision, T.J. (2013) Data reuse and the open data citation advantage. Peer J. 1, e175

3. Whitlock, M.C. (2011) Data archiving in ecology and evolution: best practices. Trends Ecol. Evol. 26, 61-65

4. Costello, M.J. et al. (2013) Biodiversity data should be published, cited and peer reviewed. Trends Ecol. Evol. 28, 454-461

5. Roche, D.G. et al. (2014) Trouble shooting public data archiving: suggestions to increase participation. PLOS Biol. 12, e10001779

6. Bloom, T. et al. (2014) Data access for the open access literature: PloS's data policy. PLoS Biol. 12, e1001797

7. Gleditsch, N.P. et al. (2003) Posting your data: will you be scooped or will you be famous? Int. Stud. Perspect. 4, 89-97

8. Caetano, D.S. and Aisenberg, A. (2014) Forgotten treasures: the fate of data in animal behaviour studies. Anim. Behav. 98, 1-5
9. Kenall, A. et al. (2014) An open future for ecological and evolutionary data? BMC Ecov. Biol. 14, 10

10. Clutton-Brock, T. and Sheldon, B.C. (2010) Individuals and populations: the role of long-term, individual-based studies of animals in ecology and evolutionary biology. Trends Ecol. Evol. 25, 562-573

11. Coulson, J.C. (1966) The influence of the pair-bond and age on the breeding biology of the kittiwake gull Rissa tridactyla. J. Anim. Ecol. 35, 269-279

12. Nisbet, I.C.T. and Dann, P. (2009) Reproductive performance of little penguins in relation to year, age, pair-bond duration, breeding date and individual quality. J. Avian Biol. 40, 296-308

13. Ryder, J.P. (1980) The influence of age on the breeding biology of colonial nesting seabirds. In Marine Birds (Behavior of Marine Animals Vol. 4) (Burger, J. et al., eds), pp. 153-168, Plenum

\section{Outstanding Questions}

How long can long-term studies continue to flourish with a requirement to publicly archive data? Providing public access to the data disadvantages those who invest time and resources in the collection and maintenance of long-term data.

Will journals and scientists with longterm studies be able to develop and implement an approach to data archiving that is acceptable to both? Collaboration, longer embargo and a code of ethics on data usage are components of a potential solution.

What are the implications of copyrighting long-term datasets? Copyrighting their datasets would allow scientists to protect their intellectual property in the same way that journals protect their publications. 
14. Genovart, M. et al. (2013) Contrasting effects of climatic variability on the demography of a trans-equatorial migratory seabird. $J$. Anim. Ecol. 82, 121-130

15. Becker, P.H. et al. (2008) Timing of initial arrival at the breeding site predicts age at first reproduction in a long-lived migratory bird. Proc. Natl. Acad. Sci. U.S.A. 105, 12349-12352

16. Reid, J.M. et al. (2010) Parentage, lifespan and offspring survival: structured variation in life history in a wild population. J. Anim. Ecol. 79, 851-862

17. Sedinger, J.S. et al. (2008) Fidelity and breeding probability related to population density and individual quality in black brant geese (Branta bernicla nigricans). J. Anim. Ecol. 77, 702-712

18. Jorgenson, J.T. et al. (1997) Effects of age, sex, disease and density of bighorn sheep. Ecology 78, 1019-1032

19. Frederiksen, M. et al. (2006) From plankton to top predators: bottom-up control of a marine food web across four tropic levels. J. Anim. Ecol. 75, 1259-1268

20. Ratcliffe, N. etal. (1998) The interactive effects of age and food supply on the breeding ecology of great skuas. J. Anim. Ecol. 67, 853-862

21. Daunt, F. et al. (2014) Longitudinal biologging reveals interplay between extrinsic and intrinsic carry over effects in a long-lived vertebrate. Ecology 95, 20177-22083

22. Mills, J.A. et al. (2008) The impact of climate fluctuation on food availability and reproductive performance of the planktivorous redbilled gull Larus novaehollandiae scopulinus. J. Anim. Ecol. 77, 1129-1142

23. Oro, D. and Furness, R.W. (2002) Influences of food availability and predation on survival of kittiwakes. Ecology 83, 2516-2528

24. Korpimäki, E. (1992) Fluctuating food abundance determines the lifetime reproductive success of male Tengmalm's owls. J. Anim Ecol. 61, 103-111

25. Festa-Bianchet, M. (1989) Individual differences, parasites, and the costs of reproduction for bighorn ewes (Ovis canadensis). J. Anim. Ecol. 58, 785-795

26. Alho, J.S. et al. (2012) No evidence for inbreeding avoidance through active mate choice in red-billed gulls. Behav. Ecol. 23, 672-675

27. Ludwig, S. and Becker, P.H. (2012) Immigration prevents inbreeding in a growing colony of a long-lived and philopatric seabird. Ibis 154, 74-84

28. Hansson, B. (2004) Marker-based relatedness predicts egg-hatching failure in great reed warblers. Conserv. Genet. 5, 339-348

29. Hayward, A.D. et al. (2013) Reproductive senescence in female Soay sheep: variation across traits and contributions of individual ageing and selective disappearance. Funct. Ecol. 27, 184-195

30. Rebke, M. et al. (2010) Reproductive improvement and senescence in a long-lived bird. Proc. Natl. Acad. Sci. U.S.A. 107, 7841-7846

31. Bonneaud, C. et al. (2006) Complex Mhc-based mate choice in a wild passerine. Proc. R. Soc. Lond. B: Biol. Sci. 273, 1111-1116

32. García-Navas, V. et al. (2009) Heterozygosity-based assortative mating in bluetits (Cyanites caeruleus): implications for evolution of mate choice. Proc. R. Soc. Lond. B: Biol. Sci. 276, 2931-2940

33. Thomson, R.L. et al. (2014) Brood size manipulations in a spatially and temporally varying environment: male Tengmalm's owls pass increased reproductive costs to offspring. Oecologia 176, 423-430

34. Riechert, J. et al. (2012) Why do experienced birds reproduce better? Possible endocrine mechanisms in a long-lived seabird, the common tern. Gen. Comp. Endocrinol. 178, 391-399
35. Bauch, C. et al. (2013) Telemere length reflects phenotypic quality and costs of reproduction in a long-lived seabird. Proc. R. Soc. B 280, 20122540

36. Bouwhuis, S. et al. (2010) Trans-generational effects on ageing in a wild bird population. J. Evol. Biol. 23, 636-642

37. Cam, E. et al. (2003) Long-term fitness consequences of early conditions in the kittiwake. J. Anim. Ecol. 72, 411-424

38. Newton, I. (1989) Lifetime Reproduction in Birds, Academic Press

39. Clutton-Brock, T.H. (1988) Reproductive Success, University of Chicago Press

40. Charmantier, A. et al. (2014) Quantitative Genetics in the Wild, Oxford University Press

41. Kruuk, L.E.B. et al. (2008) New answers for old questions: the evolutionary quantitative genetics of wild animal population. Ann Rev. Ecol. Evol. Syst. 39, 525-548

42. Charmantier, A. and Garant, D. (2005) Environmental quality and evolutionary potential: lessons from wild populations. Proc. $R$. Soc. Lond. B: Biol. Sci. 272, 1415-1425

43. Merilä, J. and Hendry, A.P. (2014) Climate change, adaptation and phenotypic plasticity: the problem and the evidence. Evol. Appl. 7 , $1-14$

44. Grant, P.R. and Grant, B.R. (2002) Unpredictable evolution in a 30-year study of Darwin's finches. Science 296, 707-711

45. Townsend, A.K. et al. (2011) Genetic monogamy across variable demographic landscapes in cooperatively breeding Florida scrubjays. Behav. Ecol. 22, 464-470

46. Teplitsky, C. et al. (2009) Heritability of fitness components in a wild bird population. Evolution 63, 716-726

47. Kruuk, L.E.B. et al. (2000) Heritability of fitness in a wild mammal population. Proc. Natl. Acad. Sci. U.S.A. 97, 698-703

48. Charmantier, A. and Gienapp, P. (2014) Climate change and timing of avian breeding and migration: evolutionary versus plastic changes. Evol. Appl. 7, 15-28

49. Teplitsky, C. and Millien, V. (2014) Climate warming and Bergmann's rule through time: is there any evidence? Evol. Appl. 7 , 156-168

50. Gienapp, P. et al. (2008) Climate change and evolution: disentangling environmental and genetic responses. Mol. Ecol. 17, $167-178$

51. Garant, D. et al. (2004) Evolution in a changing environment: a case study with great tit fledging mass. Am. Nat. 164, E115-E129

52. Cury, P.M. et al. (2011) Global seabird response to forage fish depletion-one third for the birds. Science 334, 1703-1706

53. Steen, R.G. et al. (2013) Why has the number of scientific retractions increased? PLOS ONE 8, e68397

54. Barnard, C.J. and Sibly, R.M. (1981) Producers and scroungers: a general model and its application to captive flocks of house sparrows. Anim. Behav. 29, 543-550

55. Caraco, T. and Giraldeau, L.-A. (1991) Social foraging: producing and scrounging in a stochastic environment. J. Theor. Biol. 153 , 559-583

56. Vickery, W.L. et al. (1991) Producers, scroungers, and group foraging. Am. Nat. 137, 847-863

57. Birkhead, T. (2014) Stormy outlook for long-term ecological studies. Nature 514, 7523

58. Grant, P.R. and Grant, B.R. (2014) 40 Years of Evolution: Darwin's Finches on Daphne Major Island, Princeton University Press 